moving plasma column," J. Appl. Phys., vol. 40, pp. 5066-5075, Dec. 1969.

[10] T. Shiozawa and S. Seikai, "Seattering of electromagnetic waves from an inhomogeneous magnetoplasma column moving in the axial direction," IEEE Trans. Antennas Propagat., vol. AP-20, pp. 45j-463, July 1972

[11] D. R. Wells and G. Sehmidt, "Observation of plasma rotation produced by an electrodeless plasma gun," Phys. Fluids, vol. 6, pp. 418-42I, Mar. 1963.

[12] D. L. Morse, "Plasma rotation in a hollow-cathode discharge," Phys. Fluids, vol. 8, pp. 516-521, Mar. 1965.

[13] B. Lehnert, "Rotating plasmas," Nucl. Fusion (Review Paper), vol. 11, pp. $485-533,1971$.

[14] L. Brillouin, "Theory of the magnetron. I," Phys. Rev., vol. 60, pp. 385-396, Sept. 1941.

[15] N. A. Krall and A. W. Trivelpiece, Principles of Plasma Physics. New York: MeGraw-Hill, 1973, pp. 116-118.

[16] C. V. Heer, "Resonant frequencies of an electromagnetic cavity in an accelerated system of reference," Phys. Rev., vol. 134, pp. A799-A804, May 1964.

[17] J. L. Anderson and J. W. Ryon, "Electromagnetic radiation in accelerated systems," Phys. Rev., vol. 181, pp. 1765-1775, May 1969.

[18] J. V. Bladel, "Relativistic theory of rotating disks," Proc. $I E E E$, vol. 61, pp. 260-268, Mar. 1973.

[19] T. Shiozawa, "Phenomenological and electron-theoretical study of the electrodynamics of rotating systems," Proc. IEEE, vol. 61, pp. 1694-1702, Dec. 1973.

[20] $\longrightarrow$ "Constitutive relations for a rotating electron plasma," Proc. IEEE (Letts.), vol. 62, pp. 1283-1284, Sept. 1974 .

[21] M. L. Kales, "Modes in wave guides containing ferrites," J. Appl. Phys., vol. 24, pp. 604-608, May 1953.

[22] R. C. Costen and D. Adamson, "Three-dimensional derivation of the electrodynamic jump conditions and momentum-energy laws at a moving boundary," Proc. IEEE, vol. 53, pp. 11811196, Sept. 1965.

\title{
Wave Propagation and Dipole Radiation in a Suddenly Creatèd Plasma
}

\author{
CHING-LIN JIAN'G
}

\begin{abstract}
Propagation and radiation of electromagnetic waves from oscillating sources in a suddenly created plasma are studied in this investigation. Field expressions are derived through the use of Laplace transformations. The spatial distribution of sources is taken to be arbitrary but confined.

Two cases are considered in detail: 1) plane wave propagation in a source-free region and 2) electric point dipole radiation. In the case of plane wave propagation, various aspects such as wave split, frequency shift, phase and group velocities, amplitude changes, power flows, and energy relations are discussed. In the case of electric dipole radiation, the electromagnetic fields and instantaneous radiated power are calculated and expressed in terms of Lommel functions of two variables. Asymptotic expressions and graphical results of numerical calculations of these quantities are presented. Many interesting properties of the spherical waves and power radiation are discussed.
\end{abstract}

\section{INTRODUCTION}

$\mathbf{T}$ HE STUDY of the interaction between electromagnetic radiation and plasmas having time varying parameters (for example, free electron density) has been of interest recently. The temporal variations of the plasma parameters might be produced by solar flares, by a strong laser pulse, or by a nuclear explosion. In this paper we are mainly concerned with the small amplitude waves in a suddenly created plasma. The free electron density in the medium increases suddenly from zero to some con-

Manuscript received March 1, 1974; revised July 21, 1974.

The author was with the Department of Electrical Engineering, California Institute of Technology, Pasadena, Calif. He is now with Hughes Aireraft Company, Culver City, Calif. 90230. stant value. The plasma is assumed to be isotropic, cold, lossless, homogeneous, and linear. This simple model is a useful theoretical case that could provide some insight into the basic features of wave propagation and source radiation in the presence of a time varying plasma.

The problem of wave propagation in a dielectric medium whose permittivity and permeability vary with time was studied by Morgenthaler [1]. With device application in mind many authors have also investigated the properties of waves in time varying magnetoelastic media and transmission lines [1]-[6]. Recently, using the finite difference method, Taylor, Lam, and Shumpert obtained some numerical results for pulse scattering from a perfectly conducting cylindrical rod in a cylindrical waveguide filled with time varying inhomogeneous lossy media [7]. Felsen and Whitman solved the time-domain scalar wave equation with a pulsed excitation in time varying nondispersive and dispersive media [8]; and Fante derived complicated expressions for the electric fields transmitted into a half-space with time varying properties [9]. Ginzberg studied the transition radiation in a homogeneous medium arising from a sharp change in time of the medium refractive index [10]. In these previous works, however, the problem of waves generated from oscillating sources in a time varying plasma has not been studied.

This paper presents a systematic study on the electromagnetic wave propagation and radiation from sinusoidally oscillating sources in a suddenly-created plasma. In Section II, the problem is formulated; the spatial distributions of sources are assumed to be arbitrary but con- 
fined. Originally, it is assumed that monochromatic waves are propagated and radiated. Through the use of Laplace transformations, the field equations in the suddenly-created plasma are solved in Section. III. The solutions for the electromagnetic fields are expressed in terms of their initial values and the inverse Laplace transforms of the source currents and the Green's dyadic functions. Various aspects of the wave propagation in a source-free region are then discussed in detail in Section IV. In Section V the electric and magnetic fields are calculated for an oscillating electric point dipole. The amount of power radiated by the dipole is also evaluated.

\section{FORMULATION OF THE PROBLEM}

Consider a medium, which is suddenly ionized at the time $t=0$. This suddenly-created plasma is assumed to be isotropic, cold, lossless, homogeneous, and linear. The applied source current density is specified as

$$
\mathrm{J}_{s}=\operatorname{Re}\left\{\mathrm{J}_{0}(\mathbf{r}) \exp \left(-i \omega_{0} t\right)\right\}, \quad \text { for all } t
$$

where Re is the shorthand for real part of. $J_{0}(r)$ is an arbitrary function of $\mathbf{r}$ but is confined to a finite part of space.

For $t<0$, i.e., before the plasma is created, a monochromatic wave is propagated and radiated from the source region. The electric and magnetic fields can be solved in terms of the well-known scalar and dyadic Green's functions [11].

At $t=0$ the plasma is created. It is assumed that the newly created free electrons and positive ions are stationary at the moment, that is, their velocity just after the creation $\left(t=0^{+}\right)$is zero. Thus the free current density in the medium, which is zero for $t<0$, is still zero at $t=0^{+}$. The electric and magnetic fields at $t=0^{+}$are the same as at $t=0^{-}$

$$
\begin{aligned}
& \mathbf{E}\left(\mathbf{r}, 0^{+}\right)=\mathbf{E}\left(\mathbf{r}, 0^{-}\right) \equiv \mathbf{E}_{0}(\mathbf{r}) \\
& \mathbf{H}\left(\mathbf{r}, 0^{+}\right)=\mathbf{H}\left(\mathbf{r}, 0^{-}\right) \equiv \mathbf{H}_{0}(\mathbf{r})
\end{aligned}
$$

and it follows that

$$
\left(\frac{\partial}{\partial t} \mathbf{E}\right)\left(\mathbf{r}, 0^{+}\right)=-i \omega_{0} \mathbf{E}_{0}(\mathbf{r})
$$

After $t=0$, however, the free electrons and ions in the created plasma are then set in motion by the field in the medium. It can be shown that the vector wave equation for $\mathrm{E}$, for $t>0$, is [11]

$$
\nabla \times \nabla \times \mathbf{E}+\frac{1}{c^{2}} \frac{\partial^{2}}{\partial t^{2}} \mathbf{E}+\frac{\omega_{p}^{2}}{c^{2}} \mathbf{E}=-\mu_{0} \frac{\partial}{\partial t} \mathbf{J}_{s}
$$

where $c^{2}=1 / \mu_{0} \epsilon_{0}$ and $\omega_{p} \equiv\left(N e^{2} / m \epsilon_{0}\right)^{1 / 2}$ is the plasma frequency. Equation (3) and the equation

$$
\nabla \times \mathbf{E}=-\frac{\partial}{\partial t} \mu_{0} \mathbf{H}
$$

now serve to determine $\mathbf{E}(\mathbf{r}, t)$ and $\mathbf{H}(\mathbf{r}, t)$ (for $t>0$ ), which satisfy the initial and radiation conditions. In the following section these equations will be solved for an arbitrary but confined current source distribution.

\section{GENERAL FIELD SOLUTIONS}

To solve for the field expressions, a Laplace transformation with respect to time is now performed. The results of the transformation of (3) and (4) are

$$
\nabla \times \nabla \times \hat{\mathbf{E}}(\mathbf{r}, s)+\frac{s^{2}+\omega_{p}^{2}}{c^{2}} \hat{\mathbf{E}}(\mathbf{r}, s)
$$

$$
\begin{array}{r}
=\frac{i \omega_{0} \mu_{0}}{s+i \omega_{0}} \mathbf{J}_{0}(\mathbf{r})+\frac{s-i \omega_{0}}{c^{2}} \mathbf{E}_{0}(\mathbf{r}) \\
\nabla \times \hat{\mathbf{E}}(\mathbf{r}, s)=-s \mu_{0} \hat{\mathbf{H}}(\mathbf{r}, s)+\mu_{0} \mathrm{H}_{0}(\mathbf{r})
\end{array}
$$

where we have used (1) and (2) for initial conditions. $\hat{\mathbf{E}}(\mathbf{r}, s)$ and $\hat{\mathbf{H}}(\mathbf{r}, s)$ are the Laplace transforms of $\mathbf{E}(\mathbf{r}, t)$ and $\mathbf{H}(\mathbf{r}, t)$, respectively. It is still difficult to obtain useful solutions because of the presence of the term $\left(s-i \omega_{0}\right) \mathbf{E}_{0}(\mathbf{r}) /\left(c^{2}\right)$ acting as a source that is not confined to a finite part of space. However, we are able to get rid of it by making the following appropriate transformations:

$$
\begin{aligned}
\hat{\mathbf{E}}^{\prime}(\mathbf{r}, s) & \equiv \hat{\mathbf{E}}(\mathbf{r}, s)-\frac{s-i \omega_{0}}{s^{2}+\omega_{0}^{2}+\omega_{p}^{2}} \mathbf{E}_{0}(\mathbf{r}) \\
\hat{\mathbf{H}}^{\prime}(\mathbf{r}, s) & \equiv \hat{\mathbf{H}}(\mathbf{r}, s)-\frac{s^{2}-i \omega_{0} s+\omega_{p}^{2}}{s\left(s^{2}+\omega_{0}^{2}+\omega_{p}^{2}\right)} \mathbf{H}_{0}(\mathbf{r}) \\
\hat{\mathbf{J}}^{\prime}(\mathbf{r}, s) & \equiv-\frac{i \omega_{0} \omega_{p}^{2}}{s\left(s+i \omega_{0}\right)\left(s^{2}+\omega_{0}^{2}+\omega_{p}^{2}\right)} \mathrm{J}_{0}(\mathbf{r}) .
\end{aligned}
$$

Then (20) can be reduced into

$$
\begin{gathered}
\nabla \times \nabla \times \hat{\mathbf{E}}^{\prime}(\mathbf{r}, s)+\frac{s^{2}+\omega_{p}^{2}}{c^{2}} \hat{\mathbf{E}}^{\prime}(\mathbf{r}, s)=-s \mu_{0} \hat{\mathbf{J}}^{\prime}(\mathbf{r}, s) \\
\nabla \times \hat{\mathbf{E}}^{\prime}(\mathbf{r}, s)=-s \mu_{0} \hat{\mathbf{H}}^{\prime}(\mathbf{r}, s) .
\end{gathered}
$$

Thus the retarded field solutions are [11]

$$
\begin{aligned}
& \hat{\mathbf{E}}^{\prime}(\mathbf{r}, s)=-s \mu_{0} \int_{V} \overline{\mathbf{\Gamma}}\left(\mathbf{r}, \mathbf{r}^{\prime}\right) \cdot \hat{\mathbf{J}}^{\prime}\left(\mathbf{r}^{\prime}, s\right) d V^{\prime} \\
& \hat{\mathbf{H}}(\mathbf{r}, s)=\int_{V} \nabla G\left(\mathbf{r}, \mathbf{r}^{\prime}\right) \times \hat{\mathbf{J}}^{\prime}\left(\mathbf{r}^{\prime}, s\right) d V^{\prime}
\end{aligned}
$$

where the integration with respect to the primed coordinates extends throughout the volume $V$ occupied by $\hat{\mathbf{J}}^{\prime}$. The Green's functions $G\left(\mathbf{r}, \mathbf{r}^{\prime}\right)$ and $\overline{\boldsymbol{\Gamma}}\left(\mathbf{r}, \mathbf{r}^{\prime}\right)$ are defined by

$$
G\left(\mathbf{r}, \mathbf{r}^{\prime}\right)=\frac{\exp \left[-\left|\mathbf{r}-\mathbf{r}^{\prime}\right|\left(s^{2}+\omega_{p}^{2}\right)^{1 / 2} / c\right]}{4 \pi\left|\mathbf{r}-\mathbf{r}^{\prime}\right|}
$$


and

$$
\overline{\mathbf{\Gamma}}\left(\mathbf{r}, \mathbf{r}^{\prime}\right)=\left(\overline{\mathfrak{u}}-\frac{c^{2}}{s^{2}+\omega_{p}^{2}} \nabla \nabla\right) G\left(\mathbf{r}, \mathbf{r}^{\prime}\right)
$$

where $\overline{\mathbf{u}}$ is the unit dyad [11].

The square root $\left(s^{2}+\omega_{p}^{2}\right)^{1 / 2}$ is defined to be positive for real positive $s$. For the purpose of the inversion technique the definition of the square root will be continued into the left half plane by (11)

$$
\begin{array}{r}
\left(s^{2}+\omega_{p}^{2}\right)^{1 / 2}=\left(\rho_{1} \rho_{2}\right)^{1 / 2} \exp \left[\frac{i}{2}\left(\phi_{1}+\phi_{2}\right)\right], \\
-\frac{\pi}{2} \leq \phi_{1,2} \leq \frac{3 \pi}{2} .
\end{array}
$$

Substituting (8) into (6) and performing inverse Laplace transformation we obtain the field quantities as functions of time. Hence, for $t>0$

$$
\begin{aligned}
\mathbf{E}(\mathbf{r}, t)= & \frac{\omega+\omega_{0}}{2 \omega} \mathbf{E}_{0}(\mathbf{r}) \exp (-i \omega t)+\frac{\omega-\omega_{0}}{2 \omega} \mathbf{E}_{0}(\mathbf{r}) \\
& \cdot \exp (i \omega t)+L^{-1}\left\{-s \mu_{0} \int_{V} \overline{\mathbf{\Gamma}}\left(\mathbf{r}, \mathbf{r}^{\prime}\right) \cdot \hat{\mathbf{J}}^{\prime}\left(\mathbf{r}^{\prime}, s\right) d V^{\prime}\right\}
\end{aligned}
$$$$
\mathbf{H}(\mathbf{r}, t)=\frac{\omega_{0}\left(\omega+\omega_{0}\right)}{2 \omega^{2}} \mathbf{H}_{0}(\mathbf{r}) \exp (-i \omega t)-\frac{\omega_{0}\left(\omega-\omega_{0}\right)}{2 \omega^{2}} \mathbf{H}_{0}(\mathbf{r})
$$

$$
\begin{aligned}
& \cdot \exp (i \omega t)+\frac{\omega_{p}^{2}}{\omega^{2}} \mathbf{H}_{0}(\mathbf{r}) \\
& +L^{-1}\left\{\int_{V} \nabla G\left(\mathbf{r}, \mathbf{r}^{\prime}\right) \times \hat{\mathrm{J}}^{\prime}\left(\mathbf{r}^{\prime}, s\right) d V^{\prime}\right\}
\end{aligned}
$$

where

$$
\omega=\left(\omega_{0}^{2}+\omega_{p}^{2}\right)^{1 / 2} .
$$

It can be seen that there are waves that result from the original fields at $t=0$. In (12) they are represented by those terms involving the initial values $\mathbf{E}_{0}(\mathbf{r})$ and $\mathbf{H}_{0}(\mathbf{r})$. There are also waves that result from the presence of sources in the medium. They are represented by the inverse Laplace transforms in (12). Their values are zero for $t<1 / c$ times the minimum of $\left|\mathbf{r}-\mathbf{r}^{\prime}\right|\left(\mathbf{r}^{\prime}\right.$ within $\left.V\right)$ because of the presence of $\exp \left[s t-\left|\mathbf{r}-\mathbf{r}^{\prime}\right|\left(s^{2}+\omega_{p}^{2}\right)^{1 / 2} / c\right]$ in the integrands of the inversion integrals.

Thus the electromagnetic field expressions have been derived for arbitrary but confined source distributions in a suddenly-created plasma. In the following sections plane wave propagation and electric dipole radiation will be studied in detail based on these general field expressions.

\section{PROPERTIES OF WAVE PROPAGATION}

Suppose that in a source-free region a circularly polarized plane wave is propagated in the positive $z$ direction, for $t<0$. For $t>0$, i.e., after the plasma is created, the fields are (from (12))

$$
\begin{aligned}
\mathbf{E}(\mathbf{r}, t)= & \frac{1}{\sqrt{2}}\left(\mathbf{e}_{x}+i \mathbf{e}_{y}\right) \frac{\left(1+\alpha^{2}\right)^{1 / 2}+1}{2\left(1+\alpha^{2}\right)^{1 / 2}} E_{0} \exp \left(i k_{0} z-i \omega t\right) \\
& +\frac{1}{\sqrt{2}}\left(\mathbf{e}_{x}+i \mathbf{e}_{y}\right) \frac{\left(1+\alpha^{2}\right)^{1 / 2}-1}{2\left(1+\alpha^{2}\right)^{1 / 2}} E_{0} \\
& \cdot \exp \left(i k_{0} z+i \omega t\right) \\
\mathbf{H}(\mathbf{r}, t)= & \frac{1}{2}\left(\mathbf{e}_{y}-i \mathbf{e}_{x}\right) \frac{\left(1+\alpha^{2}\right)^{1 / 2}+1}{2\left(1+\alpha^{2}\right)} H_{0} \exp \left(i k_{0} z-i \omega t\right) \\
& -\frac{1}{\sqrt{2}}\left(\mathbf{e}_{y}-i \mathbf{e}_{x}\right) \frac{\left(1+\alpha^{2}\right)^{1 / 2}-1}{2\left(1+\alpha^{2}\right)} H_{0} \\
& \cdot \exp \left(i k_{0} z+i \omega t\right)+\frac{1}{\sqrt{2}}\left(\mathbf{e}_{y}-i \mathbf{e}_{x}\right) \frac{\alpha^{2}}{1+\alpha^{2}} H_{0} \\
& \cdot \exp \left(i k_{0} z\right)
\end{aligned}
$$

where $\alpha \equiv \omega_{p} / \omega_{0}$ and $H_{0}=\left(\epsilon_{0} / \mu_{0}\right)^{1 / 2} E_{0}$.

It is clear that the original wave is split into two waves. One is propagated in the positive $z$ direction, and the other is propagated in the negative $z$ direction. The sense of the circular polarization of the two waves remains the same as the original one. The frequency of these two waves has been shifted from $\omega_{0}$ to a higher frequency $\omega=\omega_{0}\left(1+\alpha^{2}\right)^{1 / 2}$. For high frequencies, i.e., $\alpha=\omega_{p} / \omega_{0} \ll 1$, the frequency shift is

$$
\frac{\Delta \omega}{\omega_{0}}=\frac{\Delta f}{f_{0}} \cong \frac{\alpha^{2}}{2}=40.5 \frac{N}{f_{0}^{2}}
$$

where $\Delta f=\Delta \omega / 2 \pi=\left(\omega-\omega_{0}\right) / 2 \pi, f_{0}=\omega_{0} / 2 \pi$, and $N$ is the free electron density. For low frequencies, i.e., $\alpha=\omega_{p} / \omega_{0} \gg 1, \omega$ is slightly higher than the plasma frequency; the frequency shift is

$$
\frac{\Delta \omega}{\omega_{0}}=\frac{\Delta f}{f_{0}} \simeq \alpha=9 \frac{N^{1 / 2}}{f_{0}} .
$$

'The group velocity is calculated according to the formula [12]

$$
v_{a}=\left(\frac{d k}{d \omega}\right)^{-1}=\frac{c}{\left(1+\alpha^{2}\right)^{1 / 2}}
$$

which is greater for higher frequencies. As $\alpha=\omega_{p} / \omega_{0} \rightarrow 0$, $v_{0} \sim c$; as $\alpha \rightarrow \infty, v_{g} \sim 0$.

Now it is interesting to consider qualitatively what will happen when a frequency modulated pulse is propagated in a suddenly-created plasma. First, the pulse will split into two pulses; one is transmitted, the other is reflected. Second, if the original pulse has the higher frequencies in the leading portion, the transmitted pulse will be stretched out since the energy in its leading portion will propagate with a greater velocity than that in its trailing portion. However, the reflected pulse will be compressed first and stretched out subsequently, since the energy in its trailing 
portion will propagate with a greater velocity and will overtake that in its leading portion [13]-[15]. Conversely, if the original pulse has the higher frequencies in the trailing portion, the compression will take place in the transmitted pulse.

The ratios of the electric field amplitudes of the two waves to that of the original wave are

$$
\begin{aligned}
& \frac{E_{+}}{E_{0}}=\frac{\left(1+\alpha^{2}\right)^{1 / 2}+1}{2\left(1+\alpha^{2}\right)^{1 / 2}} \\
& \frac{E_{-}}{E_{0}}=\frac{\left(1+\alpha^{2}\right)^{1 / 2}-1}{2\left(1+\alpha^{2}\right)^{1 / 2}}
\end{aligned}
$$

where $E_{+}$and $E_{-}$are the amplitude of the wave propagated in the positive $z$ direction and the amplitude of the wave propagated in the negative $z$ direction, respectively. For $\alpha \ll 1, E_{+} \rightarrow E_{0}, E_{-} \rightarrow 0$; for $\alpha \gg 1, E_{+} \sim E_{-} \sim \frac{1}{2} E_{0}$. The ratios of the magnetic field amplitudes are

$$
\begin{aligned}
& \frac{H_{\leftarrow}}{H_{0}}=\frac{\left(1+\alpha^{2}\right)^{1 / 2}+1}{2\left(1+\alpha^{2}\right)} \\
& \frac{H_{-}}{H_{0}}=-\frac{\left(1+\alpha^{2}\right)^{1 / 2}-1}{2\left(1+\alpha^{2}\right)} .
\end{aligned}
$$

Both will go to zero in the low frequency limit. $\left|H_{-}\right|$is less than $1 / 8$ of the original magnitude $\left|H_{0}\right|$. It is also noted that there is a static but spatially varying magnetic field in (13). However, the slightest loss in the medium would damp out this residual static component [16].

The power flows of the waves are

$$
\begin{aligned}
& S_{+}=e_{z} \frac{\left(\left(1+\alpha^{2}\right)^{1 / 2}+1\right)^{2}}{4\left(1+\alpha^{2}\right)^{3 / 2}} S_{0} \\
& S_{-}=-e_{z} \frac{\left(\left(1+\alpha^{2}\right)^{1 / 2}-1\right)^{2}}{4\left(1+\alpha^{2}\right)^{3 / 2}} S_{0}
\end{aligned}
$$

where $S_{0}=\left(\epsilon_{0} / \mu_{0}\right)^{1 / 2}\left|E_{0}\right|^{2}$ is the original power flow. $\left|S_{-}\right|$is less than $1 / 27$ of $\left|S_{0}\right|$. In the low frequency limit the power flows for both waves are nearly zero. The net. power flow is

$$
\mathbf{S}_{\text {net }}=\mathbf{S}_{+}+\mathbf{S}_{-}=\mathbf{e}_{z} \frac{1}{1+\alpha^{2}} S_{0} .
$$

Thus the net power flow is in the positive $z$ direction and $\left|\mathbf{S}_{\text {net }}\right|<S_{0}$. For $\alpha \ll 1, S_{\text {net }} \rightarrow S_{0}$ and for $\alpha \gg 1, S_{\text {net }} \rightarrow 0$. The energy densities of the waves are [11]

$$
\begin{aligned}
w_{+} & =\frac{1}{4}\left\{\frac{\partial}{\partial \omega}\left[\omega \epsilon_{0}\left(1-\frac{\omega_{p}^{2}}{\omega^{2}}\right)\right]\right\}\left|E_{+}\right|^{2}+\frac{1}{4} \mu_{0}\left|H_{+}\right|^{2} \\
& =\frac{1}{4} \frac{\left(\left(1+\alpha^{2}\right)^{1 / 2}+1\right)^{2}}{1+\alpha^{2}} w_{0} . \\
w_{-} & =\frac{1}{4} \frac{\left(\left(1+\alpha^{2}\right)^{1 / 2}-1\right)^{2}}{1+\alpha^{2}} w_{0} .
\end{aligned}
$$

As $\alpha=\omega_{p} / \omega_{0} \rightarrow 0, w_{+} \sim w_{0}, w_{-} \sim 0$; as $\alpha \rightarrow \infty, w_{+} \sim$ $w_{-} \sim w_{0} / 4$. The total energy density of the two waves is

$$
w_{\text {tot }}=w_{+}+u_{-}=\frac{2+\alpha^{2}}{2\left(1+\alpha^{2}\right)} w_{0} .
$$

For $\alpha \ll 1, w_{\text {tot }} \rightarrow w_{0}$; for $\alpha \gg 1, w_{\text {tot }} \sim w_{0} / 2$. It is noted that $w_{\text {tot }}<w_{0}$. It can be shown that some wave energy has been transferred to static magnetic energy and kinetic energy during the creation of the plasma [16].

\section{ELECTRIC DIPOLE RADIATION}

In this section the problem of radiation from a sinusoidally oscillating electric point dipole in a suddenlycreated plasma will be considered. The origin of a spherical coordinate system is chosen to be at the dipole. The directions of the dipole and the positive $z$ axis are the same. The dipole has a moment of amplitude $p$ and sinusoidal frequency $\omega_{0}$. It is represented mathematically by

$$
\mathbf{M}(\mathbf{r}, t)=\mathbf{e}_{2} \delta(\mathbf{r}) \sin \omega_{0} t .
$$

Here $\delta(r)$ is the three-dimensional Dirac $\delta$ function. The applied source current density is related to $\mathbf{M}$ by

$$
\mathrm{J}_{s}(\mathbf{r}, t)=\frac{\partial}{\partial t} \mathbf{M}(\mathbf{r}, t)=\mathbf{e}_{\mathrm{s}} \omega_{0} p \delta(\boldsymbol{x}) \cos \omega_{0} t
$$

For $t<0$, the steady state electromagnetic fields radiated from this electric point dipole are well known [11].

For $t>0$, i.e., after the plasma is created, the fields can be obtained by taking the real part of (12)

$$
\begin{array}{r}
E_{r}(\mathbf{r}, t)=E_{r}{ }^{\prime}(\mathbf{r}, t)+\frac{1}{2}(1+X)\left\{\operatorname{Re} E_{0 r}(\mathbf{r}) \exp (-i \omega t)\right\} \\
+\frac{1}{2}(1-X) \operatorname{Re}\left\{E_{0 r}(\mathbf{r}) \exp (i \omega t)\right\} \quad(23) \\
E_{\theta}(\mathbf{r}, t)=E_{\theta}{ }^{\prime}(\mathbf{r}, t)+\frac{1}{2}(1+X) \operatorname{Re}\left\{E_{0 \theta}(\mathbf{r}) \exp (-i \omega t)\right\} \\
+\frac{1}{2}(1-X) \operatorname{Re}\left\{E_{0 \theta}(\mathbf{r}) \exp (i \omega t)\right\} \quad(24) \\
H_{\phi}(\mathbf{r}, t)=H_{\phi}{ }^{\prime}(\mathbf{r}, t)+\frac{X}{2}(1+X) \operatorname{Re}\left\{H_{0 \phi}(\mathbf{r}) \exp (-i \omega t)\right\} \\
-\frac{X}{2}(1-X) \operatorname{Re}\left\{H_{0 \phi}(\mathbf{r}) \exp (i \omega t)\right\} \quad(25)
\end{array}
$$

where $X \equiv \omega_{0} / \omega=1 /\left(1+\alpha^{2}\right)^{1 / 2} . \quad E_{0 r}(\mathbf{r}), \quad E_{0 \theta}(\mathbf{r})$, and $H_{0 \phi}(\mathbf{r})$ are the steady state amplitudes of the dipole radiation fields [11]. $E_{r}{ }^{\prime}(\mathbf{r}, t), E_{\theta}{ }^{\prime}(\mathbf{r}, t)$, and $H_{\phi}{ }^{\prime}(\mathbf{r}, t)$ are the nonzero spherical components of the inverse transforms specified in (12).

It is readily seen from (23) to (25) that, like the plane wave case, there are also two waves traveling in opposite directions. One is a spherical wave propagated outwardly from the origin, and the other is a spherical wave propagated inwardly into the origin. Their frequency is shifted from $\omega_{0}$ to $\omega=\omega_{0}\left(1+\alpha^{2}\right)^{1 / 2}$. The amplitude and power relations are the same as in the plane wave case. Moreover, the outgoing wave will vanish at a finite observation point since there is no source at the origin to radiate this wave any more; but the incoming wave will focus into 
the origin and be reflected. These novel aspects of the wave propagation are expressed mathematically in the terms $E_{\mathbf{r}}{ }^{\prime}(\mathbf{r}, t), E_{\theta}^{\prime}(\mathbf{r}, t)$, and $H_{\phi}{ }^{\prime}(\mathbf{r}, t)$. In addition to these waves, there is the radiation by the dipole, which is also included in the expressions for $E_{r}^{\prime}(\mathbf{r}, t), E_{\theta}{ }^{\prime}(\mathbf{r}, t)$, and $H_{\phi}{ }^{\prime}(\mathbf{r}, t)$.

The inverse transformations can be evaluated in terms of Lommel functions of two variables $U_{n}(u, z)$ [16], [17]. The results are

$$
\begin{aligned}
E_{\tau}^{\prime}(r, t)= & \frac{\mu_{0} \omega_{0}^{2} p}{4 \pi r} 2 \cos \theta\left\{Y \eta\left(T_{0}-\left(\xi_{0}\right)+Y \eta T_{1}{ }^{+}\left(\xi_{0}\right)\right)\right. \\
& -X \eta\left(T_{0}-(\xi)+\eta T_{1}^{+}(\xi)\right) \\
& \left.-2 \alpha Y^{2} \eta^{2} U_{1}(\gamma q, q)\right\} H\left(t-\frac{r}{c}\right) \\
E_{\theta}{ }^{\prime}(r, t)= & \frac{\mu_{0} \omega_{0}^{2} p}{4 \pi r} \sin \theta\left\{-T_{1}{ }^{+}\left(\xi_{0}\right)+Y \eta T_{0}-\left(\xi_{0}\right)\right. \\
& +Y^{2} \eta^{2} T_{1}{ }^{+}\left(\xi_{0}\right)+X\left(T_{1}+(\xi)-\eta T_{0}-(\xi)\right. \\
& \left.\left.-\eta^{2} T_{1}{ }^{+}(\xi)\right)-2 \alpha Y^{2} \eta^{2} U_{1}(\gamma q, q)\right\} H\left(t-\frac{r}{c}\right) \\
H_{\phi}{ }^{\prime}(r, t)= & \frac{\omega_{0}^{2} p}{4 \pi r c} \sin \theta\left\{-\left(1-\alpha^{2}\right)^{1 / 2} T_{1}-\left(\xi_{0}\right)\right. \\
& +\eta T_{0}{ }^{+}\left(\xi_{0}\right)+X^{2}\left(T_{1}-(\xi)-\eta T_{0}{ }^{+}(\xi)\right) \\
& \left.+2 \alpha^{2} X^{2}\left(i \alpha U_{1}(i \gamma q, q)-\eta U_{0}(i \gamma q, q)\right)\right\} \\
& \cdot H\left(t-\frac{r}{c}\right)
\end{aligned}
$$

where $Y=1 /\left(1-\alpha^{2}\right)^{1 / 2}, \eta=k_{0} r, T_{0,1} \pm(\xi) \equiv U_{0,1}(\gamma q \xi, q) \pm$ $U_{0,1}\left(\gamma q \xi^{-1}, q\right), q \equiv \omega_{p} t\left(1-\alpha^{2}\right)^{1 / 2}, \gamma \equiv(1-\beta) /(1+\beta)$, $\beta \equiv r /(c t), \xi_{0} \equiv A\left(\omega_{0}\right)$, and $A(\omega)$ is defined by

$$
\begin{array}{rlrl}
A(\omega) & =\frac{\omega+\left(\omega^{2}-\omega_{p}^{2}\right)^{1 / 2}}{\omega_{p}}, & \text { when } \omega>\omega_{p} \\
& =\frac{\omega+i\left(\omega_{p}^{2}-\omega^{2}\right)^{1 / 2}}{\omega_{p}}, \quad \text { when } \omega<\omega_{p} .
\end{array}
$$

$H(t-t / c)$ is the Heaviside step function, which is 0 when $t<r / c$, or 1 when $t \geq r / c$.

When (27) to (29) are substituted into (23) to (25), the total electric and magnetic fields $E_{r}(\mathbf{r}, t), E_{\theta}(\mathbf{r}, t)$, and $H_{\phi}(\mathbf{r}, t)$, for $t>0$, are obtained.

For small $\gamma$, corresponding to times just after the arrival of the first disturbance, the disturbing fields are

$$
\begin{aligned}
& E_{r}^{\prime}(\mathbf{r}, t)=\frac{\mu_{0} \omega_{0}^{2} p}{4 \pi r} 2 \cos \theta \frac{4}{\alpha k_{0}^{2} r^{2}} J_{3}(q) \gamma^{3} H\left(t-\frac{r}{c}\right)+0\left(\gamma^{4}\right) \\
& E_{\theta}^{\prime}(\mathbf{r}, t)=\frac{\mu_{0} \omega_{0}^{2} p}{4 \pi r} \sin \theta\left(-3+\frac{1}{k_{0}^{2} r^{2}}\right) \frac{2}{\alpha} J_{3}(q) \gamma^{3} H\left(t-\frac{r}{c}\right) \\
&+0\left(\gamma^{4}\right)
\end{aligned}
$$

$H_{\phi}{ }^{\prime}(\mathbf{r}, t)=\frac{\omega_{0}^{2} p}{4 \pi r c} \sin \theta \frac{-8}{\alpha} J_{3}(q) \gamma^{3} H\left(t-\frac{r}{c}\right)+0\left(\gamma^{4}\right)$

The amplitudes are small. The Bessel functions with arguments $q=\omega_{p} t\left(1-\beta^{2}\right)^{1 / 2}$ represent oscillations having a frequency that is initially high but continuously decreases to $\omega_{p}$.

We shall now obtain the asymptotic behavior of the total fields at a point $\mathbf{r}$ as $t \rightarrow \infty$. Through the use of the asymptotic formulas [16] we find

$$
\begin{aligned}
E_{r}(r, t) \sim & E_{p r}(r, t)+\frac{1}{2}(1-X) \\
& \cdot \operatorname{Re}\left\{E_{0 r}(\mathbf{r})(\exp (i \omega t)+\exp (-i \omega t))\right\} \\
E_{\theta}(\mathbf{r}, t) \sim & E_{p \theta}(\mathbf{r}, t)+\frac{1}{2}(1-X) \\
& \left.\cdot \operatorname{Re}\left\{E_{0 \theta}(\mathbf{r}) \exp (i \omega t)+\exp (-i \omega t)\right)\right\} \\
H_{\phi}(\mathbf{r}, t) \sim & H_{p \phi}(\mathbf{r}, t)-\frac{X}{2}(1-X) \\
& \cdot \operatorname{Re}\left\{H_{0 \phi}(\mathbf{r})(\exp (i \omega t)-\exp (-i \omega t))\right\} \\
& +\alpha^{2} X^{2}\left(\sin k_{0} r+\eta \cos k_{0} r-(\alpha+\eta)\right. \\
& \left.\cdot \exp \left(-\omega_{p} r / c\right)\right)
\end{aligned}
$$

where $E_{p r}(\mathbf{r}, t), E_{p \theta}(\mathbf{r}, t)$, and $H_{p \phi}(\mathbf{r}, t)$ are the steady-state fields of the oscillating dipole in the simple plasma medium, which are also well known [11]. The neglected terms have amplitudes vanishing faster than $t^{-1 / 2}$ as $t \rightarrow \infty$. It can be seen that radiation from the dipole has assumed its steady-state value. The outgoing wave has vanished but the incoming wave still exists because the plasma is assumed to be lossless and unbounded. The reflection of the incoming wave has also established its steady-state propagation. The incoming wave and its reflection carry the same energy but in opposite directions, hence there is no net power flow associated with these waves. It is noted that the magnetic field has a static but spatially varying component and that the electric field has a residual oscillation at the plasma frequency. Both do not contribute to the radiation and would be damped out by the slightest loss in the medium [16].

Numerical calculations of the field quantities were performed. Since the medium is isotropic, the fields were only computed in the equatorial plane $(z=0)$. In this plane only the fields $E_{\theta}$ and $H_{\phi}$ are nonzero. Figs. 1-3 show these fields as a function of the normalized time $\omega_{0} t$. The timeharmonic electric field's amplitude is denoted by horizontal dashed lines in the figures. Fig. 1 shows the fields for $\omega_{p} / \omega_{0}=0.5$ and $k_{0} r=50$, corresponding to the observation at a large distance compared with the original wavelength of the radiation. Fig. 2 corresponds to the case of dense plasma and long distance from the source with $\omega_{p} / \omega_{0}=0.9$ and $k_{0} r=50$. Fig. 3 shows the fields in an overdense plasma in which $\omega_{p} / \omega_{0}=1.2$ and $k_{0} r=5$, corresponding to the observation near the source. The beating phenomena can be seen in the fields in Figs. 1-3. In 


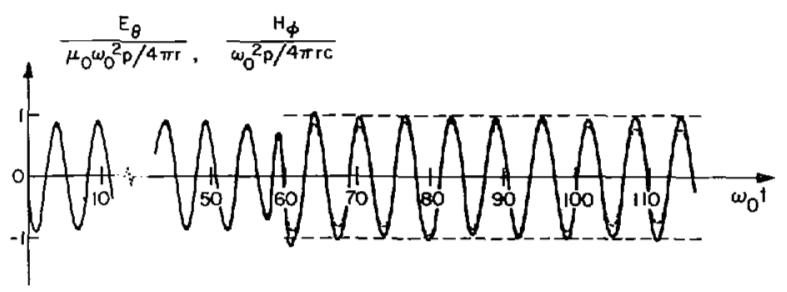

Fig. 1. $E_{\theta}(-)$ and $H_{\phi}(--)$, for $\alpha=\omega_{p} / \omega_{0}=0.5, k_{0} r=50, \theta=$ $90^{\circ}$. Horizontal dashed lines indicate time harmonic amplitude of $E_{\theta}$.

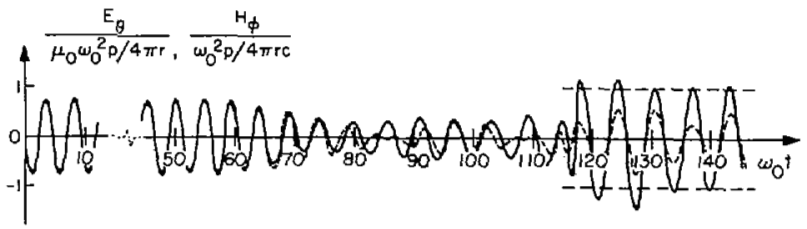

Fig. 2. $\quad E_{\theta}(-)$ and $H_{\phi}(--)$, for $\alpha=\omega_{p} / \omega_{0}=0.9, k_{0} r=50, \theta=$ $90^{\circ}$. Horizontal dashed lines indicate time harmonic amplitude of $E_{\theta}$.



Fig. 3. $E(-)$ and $H(--)$, for $\alpha=\omega_{p} / \omega_{0}=1.2, k_{0} r=5, \theta=90^{\circ}$.

Figs. 1 and 2 the effect of the group velocity on the propagation in a dispersive plasma can be seen. After $t=r / c$, i.e., $\omega_{0} t>k_{0} r$, a small disturbance comes, then the faster signal of frequency $\omega$ arrives at $\omega_{0} t \sim 50\left(1+\alpha^{2}\right)^{1 / 2}$, and finally the signal of frequency $\omega_{0}$ builds up at $\omega_{0} t \sim$ $50 /\left(1-\alpha^{2}\right)^{1 / 2}$. The time lag between these two signals is

$$
\Delta t \sim \frac{1-\left(1-\alpha^{4}\right)^{1 / 2}}{\left(1-\alpha^{2}\right)^{1 / 2}} r \text { or } \Delta\left(\omega_{0} t\right) \sim j 0 \frac{1-\left(1-\alpha^{4}\right)^{1 / 2}}{\left(1-\alpha^{2}\right)^{1 / 2}} .
$$

This phenomenon is clearly obseryed in Fig. 2 in which the ratio of the group velocity at $\omega$ to that at $\omega_{0}$ is $\left(1-(0.9)^{4}\right)^{-1 / 2} \simeq 1.7$ and the time lag is large, $\Delta\left(\omega_{0} t\right) \sim 46$.

The radiated power by the dipole for $t>0$ will now be calculated. The theory of the time-irreversible power radiated by a current distribution [18], [19] will be employed here. The power radiation formula is

$$
p^{\mathrm{rad}}(t)=-\int_{V} \mathbf{E}^{\mathrm{rad}}(\mathbf{r}, t) \cdot \mathrm{J}_{s}(\mathbf{r}, t) d \mathrm{~V}
$$

where the volume integration extends throughout the volume $V$ occupied by the source $\mathbf{J}_{s} . \mathbf{E}^{r a d}(\mathbf{r}, t)$ is the Dirac radiation field given by

$$
\mathbf{E}^{\text {rad }}(\mathbf{r}, t)=\frac{1}{2}\left(\mathbf{E}^{\text {ret }}(\mathbf{r}, t)-\mathbf{E}^{\text {adv }}(\mathbf{r}, t)\right)
$$

where $\mathbf{E}^{\text {ret }}(\mathbf{r}, t)$ and $\mathbf{E}^{\mathbf{a d v}}(\mathbf{r}, t)$ are the retarded and ad- vanced solutions for the electric field respectively. Carrying out the volume integration and the inverse integrations [16], the power radiated by the dipole for $t>0$ is

$$
\begin{aligned}
\operatorname{Prad}(t)= & \frac{\mu_{0} \omega_{0}^{4} p^{2}}{6 \pi c} \cos \omega_{0} t\left\{\frac { 1 } { ( 1 + \alpha ^ { 2 } ) ^ { 1 / 2 } } \left[\cos \left(\omega_{0}\left(1+\alpha^{2}\right)^{1 / 2} t\right)\right.\right. \\
& \left.-\left(C_{0}\left(\xi \omega_{p} t, \omega_{p} t\right)-U_{0}\left(\xi^{-1} \omega_{p} t, \omega_{p} t\right)\right)\right] \\
& \left.+\left(1-\alpha^{2}\right)^{1 / 2}\left(\zeta_{0}\left(\xi_{0} \omega_{p} t, \omega_{p} t\right)-U_{0}\left(\xi_{0}-1 \omega_{p} t, \omega_{p} t\right)\right)\right\} .
\end{aligned}
$$

For $t$ small, i.e., $\omega_{p} t \ll 1$,

$$
\begin{aligned}
P^{\mathrm{rad}}(t)= & \frac{\mu_{0} \omega_{0}^{4} p^{2}}{6 \pi c} \cos \omega_{0} t \\
& \cdot\left\{\frac{1}{\left(1+\alpha^{2}\right)^{1 / 2}} \cos \left(\omega_{0} t\left(1+\alpha^{2}\right)^{1 / 2}\right)-\frac{1}{2} \omega_{0}^{2} t^{2}\right\} \\
& +0\left(\omega_{p}^{4} t^{4}\right) .
\end{aligned}
$$

As $t \rightarrow 0^{+}$, that is, just after the plasma is created,

$P^{\mathrm{rad}}\left(0^{+}\right)=\frac{\mu_{0} \omega_{0}^{4} p^{2}}{6 \pi c} \frac{1}{\left(1+\alpha^{2}\right)^{1 / 2}}=\frac{1}{\left(1+\alpha^{2}\right)^{1 / 2}} P^{\mathrm{rad}}\left(0^{-}\right)$.

The instantaneous radiated power at $t=0^{+}$decreases to $1 /\left(1+\alpha^{2}\right)^{1 / 2}$ of its value at $t=0^{-}$because work must be done by the driving source in order to keep the dipole moment constant during the creation of the plasma.

As $t \rightarrow \infty$, we find, for $\omega_{0}>\omega_{p}$,

$$
P^{\mathrm{rad}}(t) \sim \frac{\mu_{0} \omega_{0}^{4} p^{2}}{6 \pi c}\left(1-\alpha^{2}\right)^{1 / 2} \cos ^{2} \omega_{0} t
$$

for $\omega_{0}=\omega_{p}$

$P^{\mathrm{rad}}(t) \sim \frac{\mu_{0} \omega_{0}^{4} p^{2}}{6 \pi c}\left(\frac{2}{\pi \omega_{p} t}\right)^{1 / 2} \cos \omega_{0} t \cos \left(\omega_{p} t-\frac{\pi}{4}\right) \rightarrow 0$

and for $\omega_{0}<\omega_{p}$

$$
\operatorname{Prad}(t) \sim \frac{\mu_{0} \omega_{0}^{4} p^{2}}{6 \pi c}\left(\alpha^{2}-1\right)^{1 / 2} \cos \omega_{0} t \sin \omega_{0} t .
$$

These are the power radiation formulas for an oscillating electric point dipole immersed in an isotropic, cold, lossless, homogeneous, and linear plasma. The average values are

$$
P_{\mathrm{rad}}= \begin{cases}\frac{\mu_{0} \omega_{0}^{4} p^{2}}{12 \pi c}\left(1-\alpha^{2}\right)^{1 / 2}, & \text { for } \omega_{0}>\omega_{p} \\ 0, & \text { for } \omega_{0} \leq \omega_{p} .\end{cases}
$$

Numerical calculations of the instantaneous radiated power were performed. Figs. 4-6 show the power as a function of the normalized time $\omega_{0} t$. The value $2 P^{\text {rad }}$ is denoted by horizontal dashed lines in the figures. Figs. 4 and 5 


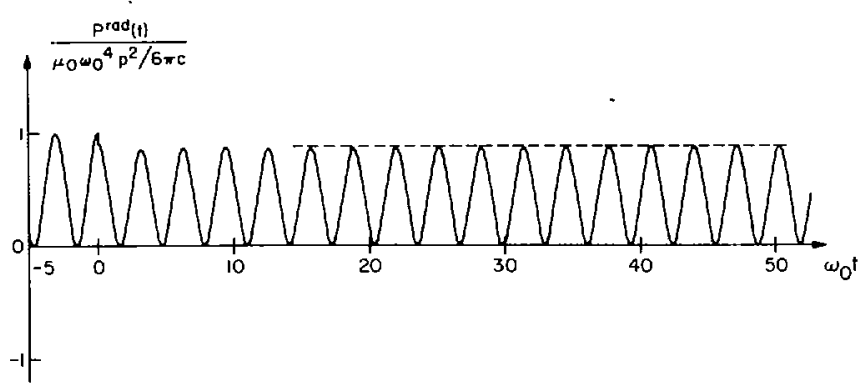

Fig. 4. $\operatorname{Prad}(t)$, for $\alpha=\omega_{p} / \omega_{0}=0.5$. Horizontal dashed lines indicate value $2 P^{\mathrm{rad}}$, where $P_{\mathrm{rad}}=\left(\mu_{0} \omega_{0}^{4} p^{2} / 12 \pi c\right)\left(1-\alpha^{2}\right)^{1 / 2}$.

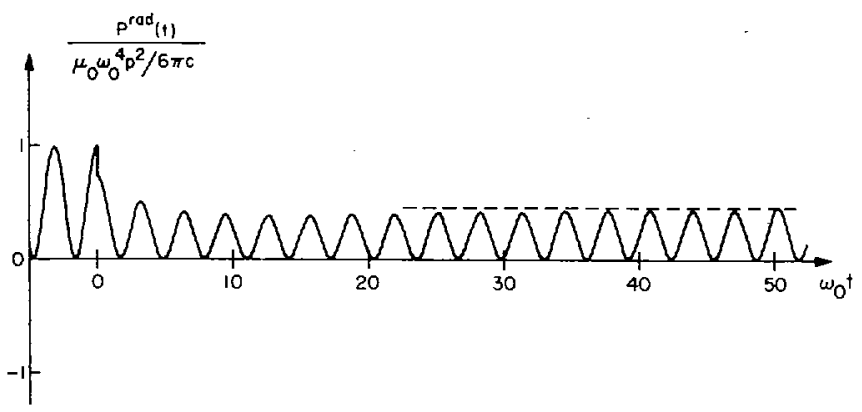

Fig. 5. $P_{\mathrm{rad}}(t)$, for $\alpha=\omega_{p} / \omega_{0}=0.9$. Horizontal dashed lines indicate value $2 P^{\mathrm{rad}}$, where $P^{\mathrm{rad}}=\left(\mu_{0} \omega_{0}^{4} p^{2} / 12 \pi c\right)\left(1-\alpha^{2}\right)^{1 / 2}$.

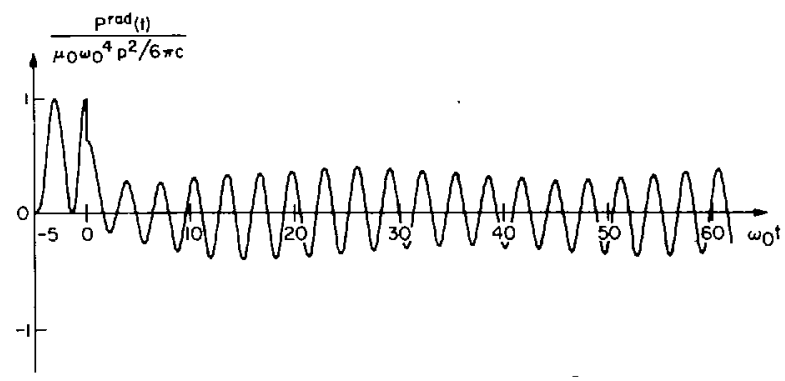

Fig. 6. $\quad P^{\mathrm{rad}}(t)$, for $\alpha=\omega_{p} / \omega_{0}=1.2$.

correspond to cases with $\omega_{p} / \omega_{0}=0.5$ and $\omega_{p} / \omega_{0}=0.9$, respectively. Fig. 6 shows the power oscillation in an overdense plasma. Beating phenomena can be seen in Figs. 4-6. In each case, a decrease in power radiation at $t=0$ is clearly observed.

\section{CONCLUSIONS}

This work studies the problem of electromagnetic wave propagation and radiation from oscillating sources in a suddenly-created plasma. Through the use of Laplace transformations, general expressions of the field quantities in the suddenly created plasma have been obtained.

In the case of plane wave propagation, it is found that there are two waves in the suddenly created plasma. One is propagated in the direction of the original wave, and the other is propagated in the opposite direction. Their frequeney is shifted from the original frequency $\omega_{0}$ to $\left(\omega_{0}^{2}+\omega_{p}^{2}\right)^{1 / 2}$, where $\omega_{p}$ is the plasma frequency. The amplitudes of the electric and magnetic fields of these two waves have been found. The power flows and energy densities have also been calculated. The net power flow of the two waves is in the direction of the original wave, but its magnitude is only $1 /\left(1+\alpha^{2}\right)$ of the original value. It is also noted that the total energy density of the two waves is less than the original one, that is, some wave energy has been lost during the creation of the plasma.

In the case of electric dipole radiation in a plasma suddenly created at $t=0$, there are also two waves for $0<t<r / c$, where $r$ is the distance between the dipole and the observation point. One is an outgoing spherical wave and the other is an incoming spherical wave. Their frequency shift, amplitudes, and power relations are the same as those in the plane wave case. At $t=r / c$, the first small disturbances arrive at $r$. Around $t \sim$ $r /\left[c /\left(1+\alpha^{2}\right)^{1 / 2}\right]$, the outgoing wave fades away, and a new wave arrives, which has the same frequency and amplitude as the incoming wave, but is propagated away from the origin. This new outgoing wave is a result of the reflection of the incoming wave at the origin. Later on, in a plasma with $\omega_{p}<\omega_{0}$, around $t \sim r /\left[c /\left(1-\alpha^{2}\right)^{1 / 2}\right]$ the radiation from the dipole builds up its steady state value. However, in an overdense plasma, the fields due to the oscillating dipole decay exponentially with distance. The exact field solutions have been obtained; and numerical calculations have been performed. The radiated power by the dipole has also been evaluated. It is found that the instantaneous radiated power suffers a decrease at the moment of plasma creation since some work must be done by the driving source to keep the dipole moment unchanged during the creation of the plasma. The power radiation gradually reaches its steady state and has the average value $\left(\mu_{0} \omega_{0}^{4} p^{2} / 12 \pi c\right)\left(1-\alpha^{2}\right)^{1 / 2}$, for $\alpha=\omega_{p} / \omega_{0}<1$ or 0 , for $\alpha \geq 1$.

\section{ACKNOWLEDGMIENT}

The author is grateful to Prof. C. H. Papas of the California Institute of Technology for his guidance and encouragement during the course of this work.

\section{REFERENCES}

[1] F. R. Morgenthaler, "Velocity modulation of electromagnetic waves," IEEE Trans. Microwave Theory Tech., vol. MTT-6, pp. 167-172, Apr. 1958.

[2] 1 . "Phase-velocity-modulated magnetoelastic waves," $J$. A ppl. Phys., vol. 37, pp. 3326-3327, 1966.

[3] B. J. Elliott and J.' B. Gunn, "Signal processing with a time varying transmission line," presented at IEEE International Solid-State Circuit Conference, Philadelphia, Pa., 1966.

[4] J. B. Gunn, "Transformation and reversal of time scale by a time-varying transmission line," Electron. Lett., vol. 2, pp. 247248,1966 .

[5] B. A. Auld, J. H. Collins, and H. R. Zapp, "Signal processing in a nonperiodically time-varying magnetoelastic medium," Proc. $I E E E$, vol. 56, pp. 258-272, Mar. 1968.

[6] S. M. Rezende and F. R. Morgenthaler, "Magnetoelastic waves in time-varying magnetic fields-I theory; II experiment," $J$. Appl. Phys., vol. 40 , pp. 524-545, 1969.

[7] C. D. Taylor, D. H. Lam, and T. H. Shumpert, "Electromagnetic pulse scattering in time-varying inhomogeneous media," IEEE Trans. Antennas Propagat., vol. AP-17, pp. 585-589, Sept. 1969.

[8] L. B. Felson and G. M. Whitman, "Wave propagation in timevarying media," IEEE Trans. Antennas Propagat., vol. AP-18, pp. 242-253, Mar. 1970. 
[9] R. L. Fante, "Transmission of electromagnetic waves into time-varying media," IEEE Trans. Antennas Propagat., vol. AP-19, pp. 417-424, May 1971.

[10] V. I. Ginzberg, "On one type of transition radiation," Izv. Vyssh. Ucheb. Zaved. Radiofiz., pp. 512-516, 1973, (in Russian).

[11] C. H. Papas, Theory of Electromagnetic Wave Propagation. New York: McGraw-Hill, 1965.

[12] J. A. Stratton, Electromagnetic Theory. New York: MeGrawHill, 1941.

[13] L. Ostrovskii, "Dispersion compression of FM waves in an inhomogeneous plasma," Radio Phys., vol. 12, pp. 1042-1046, 1964.

[14] R. E. McIntosh and S. E. El-Khamy, "Compression of transmitted pulses in plasmas," IEEE Trans. Antennas Propagat., vol. AP-18, pp. 236-241, Mar. 1970.
[15] L. B. Felsen, "Asymptotic theory of pulse compression in dispersive media," IEEE Trans. Antennas Propagat., vol. AP-19, pp. $424-432,1971$.

[16] C. L. Jiang, "Electromagnetic wave propagation and radiation in a suddenly created plasma," Ph.D. dissertation, California Institute of Technology, Pasadena, June 1972.

[17] G. N. Watson, A Treatise on the Theory of Bessel Functions. Cambridge University Press, 1966, pp. 537-550.

[18] K. S. H. Lee and C. H. Papas, "Radiation resistance and irreversible power of antennas in gyroelectric media," IEEE Trans. Antennas Propagat. (Commun.), vol. AP-13, pp. 834-835, Sept. 1965.

[19] J. J. Kenny, "Electric dipole radiation in isotropic and uniaxial plasmas," Antenna Laboratory, California Institute of Technology, Pasadena, Tech. Rep. 44, 1968.

\section{Succinct Papers}

\author{
Microstrip Antennas ${ }^{1}$ \\ JOHN Q. HOWELL
}

\begin{abstract}
Microstrip antennas consist of a planar resonant radiating element parallel to, but separated, from a ground plane by a thin dielectric substrate $(t \ll \lambda)$. These antennas are very thin and consequently rugged and easy to mount. They may be fed from the back through the ground plane or from the edge by depositing microstrip lines on the dielectric substrate. Several varieties of microstrip antennas are discussed in this paper. Design procedures are given for both linearly and circularly polarized antennas. Measured patterns are presented for antennas from UHF through $C$ band.
\end{abstract}

\section{INTRODUCTION}

In many applications involving aircraft and spacecraft where size and weight are constraints, low profile antennas may be required. In those applications where bandwidths less than a few percent are acceptable, microstrip antennas may have the desired characteristics. These antennas are constructed on a thin $(t \ll \lambda)$ dielectric sheet over a ground plane as shown in Fig. 1. The antenna element itself may be square, rectangular, round, etc., and may have more than one feed point. The antennas discussed here have either one or two feed points, but multifeed point antennas have been constructed for use at frequencies up to $K u$ band [1]-[3]. Several of the unique characteristics of microstrip antennas are listed here.

1) They are very thin and need not extend far above or below the ground plane and consequently can be made very rugged.

2) They are economical to design and construct.

3) Either linear or circular polarization is possible.

4) Dual frequency antennas can be easily constructed.

5) They are easy to mount on existing structures. Paste on antennas with a hole for the feed are conceivable.

\section{LINEARLY POLARIZED ANTENNAS}

Fig. 2 shows an $L$ band linearly polarized antenna. The actual radiating element is the large square and the smaller rectangular

Manuscript received June 18, 1973; revised August 2, 1974. The late J. Q. Howell was with the NASA Langley Research Center,

Hampton, Va. 23665 . Center, Hampton, Va. 23665 .

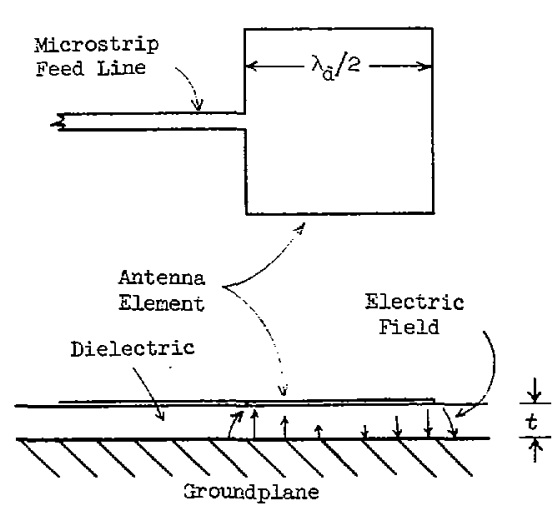

Fig. 1. Top and side view of square microstrip antenna and approximate electric field configuration.

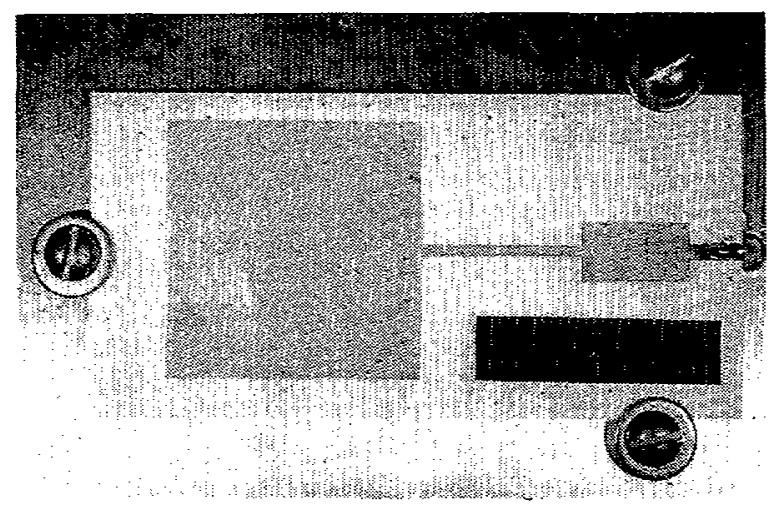

Fig. 2. $L$ band linearly polarized antenna; frequency is $1.727 \mathrm{GHz}$, $\epsilon_{r}=8.5$, dielectric thickness is $0.0625 \mathrm{in}$.

section is a quarter-wave transformer for impedance matching. The impedance transformer is necessary in order to match the $50-\Omega$ cable to the relatively high impedance at the edge of the antenna element. However, by making the radiating element rectangular it is possible to match directly to the $50 \Omega$ at the antenna input port. An antenna constructed on $\frac{1}{32}$ in Teflon fiberglass board with dimensions of $\lambda_{d} / 2$ by $\lambda_{d}\left(\lambda_{d}\right.$ is wavelength in the dielectric) had a VSWR of 1.02 when fed at the center of the long side by a $50-\Omega$ microstrip line at $S$ band. This version is currently being used in phased arrays 